\title{
EDITORIAL
}

For reprint orders, please contact: reprints@futuremedicine.com

\section{Photoacoustic mammography: prospects and promises}
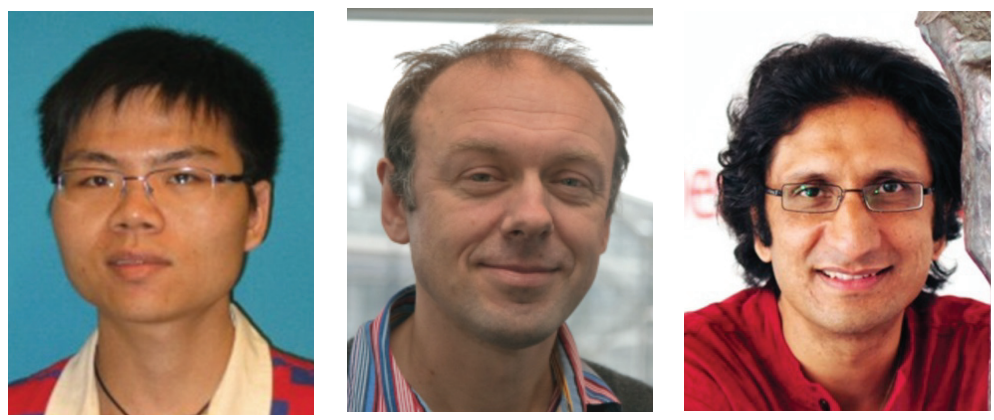

Wenfeng Xia', Wiendelt Steenbergen' ${ }^{1}$ \& Srirang Manohar ${ }^{*, 1}$

Breast cancer is the most frequently occurring malignancy in women, and the leading cause of cancer death, with up to 0.5 million mortalities in 2008 [1]. The strategy to reduce breast cancer deaths is by early detection, reliable diagnosis and effective treatments. The standard imaging modality for breast cancer detection by screening asymptomatic women is $\mathrm{x}$-ray imaging. Diagnosis is performed after the triple assessment of x-ray mammography, ultrasonography and biopsy. MRI is used in specific problem-solving situations, such as when contradictory results are obtained from x-ray and ultrasound images. $\mathrm{x}$-ray mammography is capable of producing $2 \mathrm{D}$ projection images with a high spatial resolution. However, $\mathrm{x}$-ray mammography, besides possessing ionizing hazards and requiring painful breast compression, is less sensitive in dense breasts [2] Ultrasonography is limited by poor soft tissue contrast, inherent speckle noise, strong operator dependence and lack of standardization. Both x-ray mammography and ultrasound imaging focus on morphological changes in tissue, which are usually manifested in later progression of disease.
MRI has high sensitivity, being capable of visualizing functional information of tumors, but suffers from variable specificity, a relatively high cost and the need of a contrast agent, and excludes pregnant women, subjects with metal implants and claustrophobia [2]. Thus, there is a great need for an alternative nonionizing imaging technique to detect and diagnose early stages of breast cancer with high sensitivity and specificity, but without discomfort or the need of contrast agents.

Methods that can potentially address the limitations associated with conventional imaging modalities are those that use light. The vascularization associated with cancer leads to an optical absorption contrast compared with healthy tissue. Optical imaging with its nonionizing nature thus potentially has a high sensitivity due to the rich optical absorption contrast and a high specificity due to the ability to perform spectroscopy. However, the resolution of optical mammography is poor due to strong light scattering in biological tissues. Photoacoustic (PA; also called optoacoustic) imaging addresses the resolution limitation of optical imaging

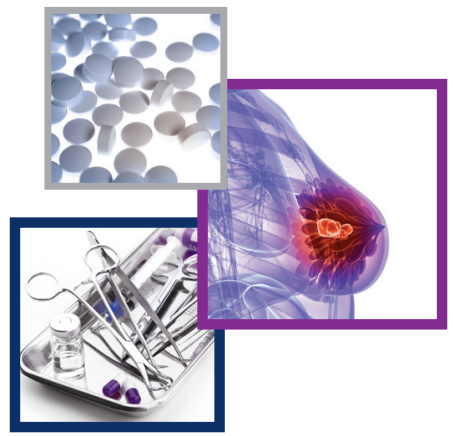

\section{KEYWORDS}

- hybrid imaging • optical contrast $\bullet$ optoacoustic imaging - photoacoustic imaging $\bullet$ tumor vascularization 
“...photoacoustic imaging combines the advantages from optical imaging, namely a high vascularization-driven optical absorption contrast from cancer, and the advantages from ultrasound imaging, namely a high resolution and a large imaging depth.” by allowing optical absorbing chromophores to emit ultrasound (US) waves. This occurs by thermal-elastic expansion at absorbing sites in response to short pulsed light excitation. Instead of detecting light, PA imaging detects US waves at the tissue surface. Since US scattering is considerably lower than light scattering in tissue, PA imaging offers superior resolutions.

Thus, PA imaging combines the advantages from optical imaging, namely a high vascularization-driven optical absorption contrast from cancer, and the advantages from ultrasound imaging, namely a high resolution and a large imaging depth. Early studies have shown much promise in visualizing breast cancer [3-10].

The application of PA using near infrared (NIR) light excitation for breast imaging was introduced by Oraevsky [3] and Kruger [4]. Oraevsky et al. reported an early 2D imager called the laser optoacoustic imaging system (LOIS), and the first results were reported in 2001 [6]. In 2004, Manohar et al. presented the Twente Photoacoustic Mammoscope (PAM), which produces $3 \mathrm{D}$ images in regions of interest [5], and first experiences of NIR-PA imaging of breast cancer in human subjects were reported in 2007 [6]. In this work, in four of the five cases of malignancies studied, PAM images revealed regions possessing higher PA intensity than the surrounding background, which was attributed to tumor angiogenesis. In 2009, Oraevsky et al. presented results using an upgraded version of their 2D imager (LOIS-64) imaging 27 patients [7]. They were able to visualize 18 out of 20 malignant lesions. In 2012, the author's group showed new results on 12 patients using PAM, corroborating the earlier studies and giving new insights. A significant finding was that PAM could successfully visualize malignancies with a superior imaging contrast to $\mathrm{x}$-ray mammography [8], and the contrast was found to be independent of fibroglandular breast density. In the same year, Kyoto University [9] performed clinical studies on 27 patients using a prototype system based on a detection geometry similar to PAM, and found 20 out of 27 breast cancer lesions to be visible. In 2013, the group of Kruger reported a dedicated 3D breast imager. They were able to visualize vasculature throughout the breast tissue, including close to the chest wall in eight breasts from four healthy volunteers [10].

While much still needs to be learned, the clinical case studies presented to date have been very promising, and with this there is impetus to improve the present imaging prototypes. Here we present our thoughts on the future directions of technological development of PA mammography.

\section{Sensitive \& faithful detection of ultrasound}

Imaging at large depths $(5+\mathrm{cm})$, which is required in the breast, represents a great challenge mainly due to the strong light attenuation in breast tissue, which causes low signal amplitudes to be produced. Furthermore, since the expected PA signals have broad frequency spectra ranging from tens of $\mathrm{kHz}$ to a few $\mathrm{MHz}$, a broadband detector is required. Therefore, the challenge ahead lies in researching and developing sensitive and faithful ultrasound detectors, a necessity that requires advanced and optimized piezoelectric transducers, or altogether new ultrasound detectors based on optical means [11]. The authors' group has developed a laboratory prototype tomography system with an optimized piezoelectric ultrasound detector array possessing unprecedented sensitivity with tolerable bandwidth, which could pave the way for sensitive detection of breast cancer [12-14].

\section{Multispectral PA mammography}

Multispectral optoacoustic (PA) tomography (MSOT) has been used to resolve chromophoric and fluorescent agents with molecular specificity [15]. This technique utilizes multispectral illumination to differentiate specific spectral signatures of chromophores or key reporter agents over the background tissue absorption by decomposing the spectral contributions of different photon-absorbers. Multispectral PA mammography can benefit the differentiation of the abundance of specific tissue components in the breast and the quantification of blood oxygen saturation, and thus improve the specificity. However, complex tissue structures in the breast could challenge the estimation of light fluence distribution, a necessary knowledge used to quantify the optical absorption coefficients of different photon absorbers at distinct light wavelengths in MSOT. Various inversion algorithms have been formulated attempting to recover the absorption coefficient in an iterative framework. Here the error between the measurement and its computed counterpart calculated using assumed optical properties is iteratively minimized using various algorithms. A novel experimental method also holds promise to achieving quantitative PA mapping of chromophore concentrations [16]. The approach combines 
PAs with acousto-optic modulation of light using focused ultrasound. Early work has shown that PA signals of absorbing insertions in a phantom at different depths can be fluence-corrected by this method to achieve an accuracy of 5\% in estimation of absorption coefficient.

\section{Multimodality imaging}

PA mammography can be combined with other imaging modalities to further improve specificity. The application of radiofrequency (RF) or microwave excitations can also generate ultrasound in the breast. This method, called thermoacoustic (TA) tomography, was introduced by Kruger et al. [5]. The absorbed RF energy excites US pressure waves just as in PA. Since $\mathrm{RF}$ radiation can penetrate tens of centimeters tissues due to its long wavelength, TA tomography is expected to provide larger imaging depths than PA. TA tomography can be easily integrated with PA tomography with the two modalities sharing the same US detection and image reconstruction. This combination when applied in breast imaging has the potential to further improve the imaging sensitivity at large depths. The group of Wang integrated both PA and TA tomography in a single system in 2008 [17]. Furthermore, PA tomography can be combined with US tomography, since they share the same US detection [18]. The combination of PA and US tomography will have additional clinical values compared with single PA modality. US reflection imaging provides the breast anatomy and maps the tissue changes in echogenicity. It is also expected that speed of sound (SOS) and acoustic attenuation (AA) mapping provides additional diagnostic value, since higher SOS and $A A$ values are reported to be associated with tumor mass compared with normal breast tissue.

\section{PA mammography systems with handheld probes}

The abovementioned systems are dedicated imaging systems where the subject lies on a bed, which is the patient-instrument interface. However, these systems are usually bulky and expensive, which require custom-made ultrasound detection systems. PA mammography systems could also be built around flexible hand-held probes, which could have application in diagnosis. A straightforward approach is to integrate a pulsed laser source to a commercial US array as reported by Kolkman et al. [19]. Compared to the above-discussed CT-based breast imagers, the use of commercial US arrays has distinct advantages. First, the system integrating both PA and US imaging provides additional information to purely PA imaging. Second, PA mammography performed simultaneously with a routinely used ultrasonography will pave the way for the acceptance of PA mammography by medical staff. Finally, the use of commercial US arrays will shorten the time for system development and thus reduce the system cost.

\section{Contrast agents enhanced PA mammography}

Most of current PA mammography systems are based on hemoglobin detection, utilizing endogenous chromophores. The detection of the extremely weak PA signals from small cancers deep inside the breast challenges the sensitivity of the imaging systems. Exogenous contrast agents can be used to greatly enhance the imaging contrast, and further allow cellular and molecular imaging for enhanced specificity of PA imaging [20].

Along with the continuous growth of the PA research community, more and more interesting functions of PA imaging will be discovered and applied to breast imaging. The future roles of PA mammography in clinical practice will strongly depend on the outcome of clinical case studies. Sensitivity and specificity of the imagers need to be evaluated in comparison to x-ray mammography and MRI. With tremendous promise, PA mammography could supplement $\mathrm{x}$-ray mammography for use as a diagnosis tool, or if possessing sufficient sensitivity and specificity, could potentially replace x-ray mammography for breast cancer screening. We look forward to seeing the commercialization of PA mammography in the near future.

Financial \& competing interests disclosure

The financial support from the Agentschap NL InnovationOriented Research Programmes Photonic Devices under the HYMPACT Project (IPD083374) and High Tech Health Farm, the Province Overijssel, are gratefully acknowledged. W Steenbergen and S Manohar have minority share in $P A$ Imaging $B V$, which did not financially support the research. The authors have no other relevant affiliations or financial involvement with any organization or entity with a financial interest in or financial conflict with the subject matter or materials discussed in the manuscript apart from those disclosed.

No writing assistance was utilized in the production of this manuscript. 


\section{References}

1 Jemal A, Siegel R, Ward E et al. Cancer statistics, 2008. CA Cancer J. Clin. 58(2), 71-96 (2008).

2 Berg WA, Gutierrez L, Nessaiver MS et al. Diagnostic accuracy of mammography, clinical examination, US, and MR imaging in preoperative assessment of breast cancer. Radiology 233, 830-849 (2004).

3 Oraevksy AA, Karabutov AA, Solomatin SV et al. Laser optoacoustic imaging of breast cancer in vivo. Proc. SPIE 6 (2001).

4 Kruger R A, Kiser WK, Romilly JAP, Schmidt P. Thermoacoustic CT of the breast: pilot study observations. Proc. SPIE 1 (2001).

5 Manohar S, Kharine A, van Hespen JCG, Steenbergen W, Van Leeuwen TG.

Photoacoustic mammography laboratory prototype: imaging of breast tissue phantoms. J. Biomed. Opt. 9, 1172-1181 (2004).

6 Manohar S, Vaartjes SE, Van Hespen JCG et al. Initial results of in vivo noninvasive cancer imaging in the human breast using near-infrared photoacoustics. Opt. Express 15, 12277-12285 (2007).

7 Ermilov SA, Khamapirad T, Conjusteau A et al. Laser optoacoustic imaging system for detection of breast cancer. J. Biomed. Opt. 14(2), 024007 (2009).
8 Heijblom M, Piras D, Xia W et al. Visualizing breast cancer using the Twente photoacoustic mammoscope: what do we learn from twelve new patient measurements? Opt. Express 20, 11582-11597 (2012).

9 Kitai T, Torii M, Sugie T et al. Photoacoustic mammography: initial clinical results. Breast Cancer 21(2), 146-153 (2014).

10 Kruger RA, Kuzmiak CM, Lam RB, Reinecke DR, Del Rio SP, Steed D. Dedicated 3D photoacoustic breast imaging. Med. Phys. 40, 113301 (2013).

11 Zhang E, Laufer J, Beard P. Backward-mode multiwavelength photoacoustic scanner using a planar Fabry-Perot polymer film ultrasound sensor for high-resolution three-dimensional imaging of biological tissues. Appl. Opt. 47(4), 561-577 (2008).

12 Xia W, Piras D, van Hespen JCG et al. An optimized ultrasound detector for photoacoustic breast tomography. Med. Phys. 40(3), 032901 (2013).

13 Xia W, Piras D, van Hespen JCG, Steenbergen W, Manohar S. A new acoustic lens material for large area detectors in photoacoustic breast tomography. Photoacoustics 1(2), 9-18 (2013).

14 Xia W, Piras D, Singh MKA et al. Design and evaluation of a laboratory prototype system for $3 \mathrm{D}$ photoacoustic full breast tomography.
Biomed. Opt. Express 4(11), 2555-2569 (2013).

15 Razansky D, Distel M, Vinegoni C et al. Multispectral opto-acoustic tomography of deep-seated fluorescent protein it in vivo. Nat. Photonics 3, 412-417 (2009).

16 Daoudi K, Hussain A, Hondebrink E, Steenbergen W. Correcting photoacoustic signals for fluence variations using acoustooptic modulation. Opt. Express. 20(13), 14117-14129 (2012).

17 Pramanik M, Ku G, Li C, Wang LV. Design and evaluation of a novel breast cancer detection system combining both thermoacoustic (TA) and photoacoustic (PA) tomography. Med. Phys. 35, 2218-2223 (2008).

18 Jose J, Willemink RGH, Resink S et al. Passive element enriched photoacoustic computed tomography (PER PACT) for simultaneous imaging of acoustic propagation properties and light absorption. Opt. Express, 19(3), 2093-2104 (2011).

19 Kolkman RGM, Brands PJ, Steenbergen W, van Leeuwen TG. Real-time in vivo photoacoustic and ultrasound imaging. J. Biomed. Opt. 13(5), 050510 (2008).

20 Ku F, Wang LV. Deeply penetrating photoacoustic tomography in biological tissue enhanced with an optical contrast agent. $O p t$. Lett. 30(5), 507-509 (2005). 